\title{
Oxaliplatin-Induced Hyperammonemic Encephalopathy in a Patient with Metastatic Pancreatic Cancer: A Case Report
}

\author{
Takatsugu Ogata Hironaga Satake Misato Ogata Yukimasa Hatachi \\ Hisateru Yasui
}

Department of Medical Oncology, Kobe City Medical Center General Hospital, Kobe, Japan

\section{Keywords}

Hyperammonemia · Oxaliplatin - GEMOX · Pancreatic cancer · Urea cycle $\cdot$ Hyperammonemic encephalopathy

\section{Abstract}

Oxaliplatin-based chemotherapy is widely used to treat advanced cancer. Oxaliplatin-induced hyperammonemic encephalopathy is rarely reported. Here, we report a case of oxaliplatininduced hyperammonemic encephalopathy occurring after gemcitabine plus oxaliplatin (GEMOX) chemotherapy in a patient with pancreatic cancer. A 76-year-old man received GEMOX regimen as first-line treatment for pancreatic adenocarcinoma with peritoneal dissemination. GEMOX consists of gemcitabine $\left(1,000 \mathrm{mg} / \mathrm{m}^{2}\right)$ and oxaliplatin $\left(100 \mathrm{mg} / \mathrm{m}^{2}\right)$ on day 1 , repeated every 2 weeks. The second cycle of GEMOX was administered as planned. However, he appeared to have difficulties with daily activities. Two days later, he visited the emergency room complaining of drowsiness. On examination, the patient had a Glasgow Coma Scale (GCS) score of 14 (E4V4M6), and asterixis was not present. Blood examination 


\section{Case Reports in Oncology}

revealed a serum ammonia level of $202 \mu \mathrm{g} / \mathrm{dL}$. The levels of serum hepatic enzymes were only mildly elevated, and the hemoglobin level was typical for this patient. Computed tomography, magnetic resonance imaging, lumbar puncture test, and blood culture showed no abnormality. Based on these results, he was diagnosed with oxaliplatin-induced hyperammonemia. One day after hospitalization, GCS score had significantly decreased to 6 (E1V1M4). His consciousness disorder improved after administration of a nutritional supplement containing a high concentration of branched-chain amino acids for 5 days, and the level of serum ammonia improved to $79 \mu \mathrm{g} / \mathrm{dL}$. He stated that he could not remember the episode. The findings of this case suggest the importance of examining serum ammonia levels in patients receiving an oxaliplatin-containing regimen who develop disordered consciousness.

\section{Introduction}

Oxaliplatin-based chemotherapy is widely used to treat advanced colorectal, gastric, and pancreatic cancer. The common side effects related to oxaliplatin are nausea, vomiting, bone marrow suppression, and peripheral neuropathy [1]. Oxaliplatin-induced hyperammonemic encephalopathy is rarely reported. Hyperammonemic encephalopathy is a rare complication that can occur after oxaliplatin-based chemotherapy, and it manifests as altered mental status without any radiologic abnormality or abnormal laboratory test results, except for elevated serum ammonia levels. Herein, we report a case of oxaliplatin-induced hyperammonemic encephalopathy that occurred after gemcitabine plus oxaliplatin (GEMOX) chemotherapy in a patient with pancreatic cancer.

\section{Case Report}

A 76-year-old man with no history of hepatitis B virus or hepatitis C virus infection had pancreatic adenocarcinoma with peritoneal dissemination. The clinical stage was IV (cT3N1M1). The main site of metastasis was the peritoneum, and there was no liver metastasis. With respect to his family history, his father had been diagnosed with pancreatic cancer. The level of serum carcinoembryonic antigen was $87.6 \mathrm{ng} / \mathrm{dL}$ (reference value, $<5.0$ $\mathrm{ng} / \mathrm{dL}$ ) and that of serum carbohydrate antigen $19-9$ was $15,030 \mathrm{U} / \mathrm{mL}$ (reference value, $<37.0 \mathrm{U} / \mathrm{mL}$ ). The patient received a GEMOX regimen as first-line therapy as part of a clinical trial. GEMOX consists of gemcitabine $\left(1,000 \mathrm{mg} / \mathrm{m}^{2}\right)$ and oxaliplatin $\left(100 \mathrm{mg} / \mathrm{m}^{2}\right)$ on day 1 , and administration is repeated every 2 weeks. The second cycle of GEMOX was administered as planned. However, the patient appeared to have difficulty performing daily activities on the night of admission. Two days later (day 3), he visited the emergency room complaining of drowsiness. His body temperature, pulse rate, and blood pressure were $37.1^{\circ} \mathrm{C}, 80$ beats per min, and 133/74 mm Hg, respectively. His Glasgow Coma Scale (GCS) score was 14 (E4V4M6). Upon examination, asterixis was not present, nor were dehydration or constipation. Blood examination revealed a serum ammonia level of $202 \mu \mathrm{g} / \mathrm{dL}$ (reference value, 19$54 \mu \mathrm{g} / \mathrm{dL}$ ). Levels of serum hepatic enzymes were mildly elevated (aspartate aminotransfer- 


\section{Case Reports in Oncology}

ase, $101 \mathrm{IU} / \mathrm{L}$ [reference value, 8-40 IU/L]; alanine aminotransferase, $69 \mathrm{IU} / \mathrm{L}$ [reference value, 8-40 IU/L]). Serum hemoglobin level was $12.8 \mathrm{~g} / \mathrm{dL}$ (reference value, 13.4-17.6 g/dL) and it had not decreased compared with the usual level for this patient. Serum sodium, serum albumin, and serum cholinesterase levels were $136 \mathrm{mEq} / \mathrm{L}$ (reference value, 136-148 $\mathrm{mEq} / \mathrm{L}$ ), $3.8 \mathrm{~g} / \mathrm{dL}$ (reference value, 3.9-4.9 g/dL), and $124 \mathrm{U} / \mathrm{L}$ (reference value, 165-470 U/L), respectively. Serum C-reactive protein and serum white blood cell count were 0.09 $\mathrm{mg} / \mathrm{dL}$ (reference value, $0-0.5 \mathrm{mg} / \mathrm{dL}$ ) and $6,000 / \mu \mathrm{L}$ (reference value, 3,900-9,000/ $\mu \mathrm{L}$ ), respectively. Serum blood urea nitrogen and serum creatinine levels were $24.5 \mathrm{mg} / \mathrm{dL}$ (reference value, $8.0-20.0 \mathrm{mg} / \mathrm{dL}$ ) and $0.58 \mathrm{mg} / \mathrm{dL}$ (reference value, $0.60-1.10 \mathrm{mg} / \mathrm{dL}$ ), respectively. Computed tomography revealed no liver metastasis or splenomegaly, but there was collateral circulation due to portal vein stenosis. Magnetic resonance imaging, cerebrospinal fluid examination, and blood culture revealed no abnormalities.

Based on these results, the patient was diagnosed with oxaliplatin-induced hyperammonemia. One day after hospitalization, his GCS score decreased to 6 (E1V1M4), which was his worst value. His consciousness disorder improved after administration of a nutritional supplement containing a high concentration of branched-chain amino acids for 5 days, and his level of serum ammonia improved to $79 \mu \mathrm{g} / \mathrm{dL}$. The patient was discharged 13 days later and hyperammonemia has not recurred despite the absence of predictive medication. We considered hyperammonemic encephalopathy was a severe adverse event of chemotherapy and thus ceased administration of this regimen in his case.

\section{Discussion}

Currently, FOLFIRINOX (5-fluorouracil [5-FU], oxaliplatin, and irinotecan) and gemcitabine plus nab-paclitaxel are standard therapies for metastatic pancreatic adenocarcinoma. However, many clinical trials have used other regimens, including GEMOX, gemcitabine plus cisplatin, or gemcitabine plus S-1 [2-4]. Especially in the case of patients who have a family history of either breast, ovarian, or pancreatic cancers, chemotherapy including a platinum agent is associated with a better survival rate [5]. In this case, the patient had a family history of pancreatic cancer. The FOLFIRINOX regimen is not recommended for patients aged $>76$ years because they are typically excluded from clinical trials, and the adverse events caused by this regimen can be severe [6]. Our patient received GEMOX because he participated in a clinical trial.

Hyperammonemic encephalopathy is uncommon, and 5-FU, cytarabine, and vincristine are known to be associated with this adverse event [7]. Hyperammonemic encephalopathy is a severe adverse event of oxaliplatin, and its frequency is reported to be $0.07 \%$ when oxaliplatin is used in combination with 5-FU. In contrast, gemcitabine has not been reported to be associated with hyperammonemic encephalopathy. It is possible that, in this case, the patient actually experienced gemcitabine-induced hyperammonemia; however, based on previous reports, we concluded that this was a case of oxaliplatin-induced hyperammonemic encephalopathy.

The risk factors for chemotherapy-induced hyperammonemia are dehydration, constipation, renal dysfunction, infection, or catabolism of skeletal muscle due to malnutrition [8]. However, our patient did not have any obvious risk factors. One study reported a case of 


\section{Case Reports in Oncology}

hyperammonemia due to collateral circulation, and, in this case, collateral circulation was identified by computed tomography [9]. Hyperammonemic encephalopathy due to collateral circulation usually shows recurrence [10]. Our patient did not develop recurrent hyperammonemia; hence, we concluded that collateral circulation was not the cause of this event.

The findings of our case indicate the importance of analyzing serum ammonia levels in patients receiving an oxaliplatin-containing regimen who develop disordered consciousness. In cases of chemotherapy-induced hyperammonemic encephalopathy, one suggested mechanism includes a catabolic state induced by chemotherapeutic agents that overwhelm the capacity of the urea cycle [11]. The mechanism of action of oxaliplatin involves the mitochondrial pathway; hence, it is possible that oxaliplatin impairs the urea cycle [12]. To identify whether oxaliplatin impairs the activity of the urea cycle enzyme, it is effective that the laboratory tests of plasma amino acid and urinary orotic acid are performed. When the activity of the urea cycle enzyme is impaired, plasma amino acid levels reveal low ornithine with normal alanine and glutamine, and the urinary orotic acid level is elevated [13]. While cisplatin-related hyperammonemia has been reported, cisplatin was administered only in combination with 5-FU in previous studies [14]. To our knowledge, no study has reported a case of carboplatin-associated hyperammonemia. The half-life of oxaliplatin is longer than that of other platinum-based agents (Table 1) [15]. It is hypothesized that, because oxaliplatin persists for a longer time than other platinum agents, hyperammonemia does not occur with cisplatin or nedaplatin, but does with oxaliplatin.

\section{Conclusion}

We suggest that oxaliplatin-induced hyperammonemia is caused by a disturbance of the urea cycle. Further studies should be undertaken to clarify the mechanism involved.

\section{Statement of Ethics}

The Ethics Committee of Kobe City Medical Center General Hospital approved the study.

Written informed consent was obtained from the deceased's wife for publication of this case report.

\section{Disclosure Statement}

The authors report no conflict of interest.

\section{References}

1 DeVita VT, Lawrence TS, Rosenberg SA: DeVita, Hellman, and Rosenberg's Cancer Principles \& Practice of Oncology. Philadelphia, Wolters Kluwer, 2015. 
H, Holstege A, Hinke A, Schalhorn A, Wilkowski R: Randomized phase III trial of gemcitabine plus cisplatin compared with gemcitabine alone in advanced pancreatic cancer. J Clin Oncol 2006;24:39463952.

- Louvet C, Labianca R, Hammel P, Lledo G, Zampino MG, Andre T, Zaniboni A, Ducreux M, Aitini E, Taieb J, Faroux R, Lepere C, de Gramont A; GERCOR, GISCAD: Gemcitabine in combination with oxaliplatin compared with gemcitabine alone in locally advanced or metastatic pancreatic cancer: results of a GERCOR and GISCAD phase III trial. J Clin Oncol 2005;23:3509-3516.

-4 Ueno H, Okusaka T, Furuse J, Yamao K, Funakoshi A, Boku N, Ohkawa S, Yokosuka O, Tanaka K, Moriyasu F, Nakamori S, Sato T: Multicenter phase II study of gemcitabine and S-1 combination therapy (GS therapy) in patients with metastatic pancreatic cancer. Jpn J Clin Oncol 2011;41:953-958.

5 Fogelman D, Sugar EA, Oliver G, Shah N, Klein A, Alewine C, Wang H, Javle M, Shroff R, Wolff RA, Abbruzzese JL, Laheru D, Diaz LA Jr: Family history as a marker of platinum sensitivity in pancreatic adenocarcinoma. Cancer Chemother Pharmacol 2015;76:489-498.

-6 Conroy T, Desseigne F, Ychou M, Bouche O, Guimbaud R, Becouarn Y, Adenis A, Raoul JL, GourgouBourgade S, de la Fouchardiere C, Bennouna J, Bachet JB, Khemissa-Akouz F, Pere-Verge D, Delbaldo C, Assenat E, Chauffert B, Michel P, Montoto-Grillot C, Ducreux M; Groupe Tumeurs Digestives of Unicancer; PRODIGE Intergroup: FOLFIRINOX versus gemcitabine for metastatic pancreatic cancer. N Engl J Med 2011;364:1817-1825.

7 Chang YY, Lin JK, Jiang JK: Oxaliplatin-related hyperammonaemic encephalopathy in a patient with colon cancer. Colorectal Dis 2012;14:e821.

8 Yi HJ, Hong KS, Moon N, Chung SS, Lee RA, Kim KH: Acute hyperammonemic encephalopathy after 5fluorouracil based chemotherapy. Ann Surg Treat Res 2016;90:179-182.

-9 Sherlock S, Summerskill WH, White LP, Phear EA: Portal-systemic encephalopathy; neurological complications of liver disease. Lancet 1954;267:454-457.

10 Inoue H, Emori K, Toyonaga A, Oho K, Kumamoto M, Haruta T, Mitsuyama K, Tsuruta O, Sata M: Long term results of balloon-occluded retrograde transvenous obliteration for portosystemic shunt encephalopathy in patients with liver cirrhosis and portal hypertension. Kurume Med J 2014;61:1-8.

11 Lazier J, Lupichuk SM, Sosova I, Khan AA: Hyperammonemic encephalopathy in an adenocarcinoma patient managed with carglumic acid. Curr Oncol 2014;21:e736-e739.

12 Gourdier I, Del Rio M, Crabbe L, Candeil L, Copois V, Ychou M, Auffray C, Martineau P, Mechti N, Pommier Y, Pau B: Drug specific resistance to oxaliplatin is associated with apoptosis defect in a cellular model of colon carcinoma. FEBS Lett 2002;529:232-236.

13 Chapuy CI, Sahai I, Sharma R, Zhu AX, Kozyreva ON: Hyperammonemic encephalopathy associated with fibrolamellar hepatocellular carcinoma: case report, literature review, and proposed treatment algorithm. Oncologist 2016;21:514-520.

14 Kim YA, Chung HC, Choi HJ, Rha SY, Seong JS, Jeung HC: Intermediate dose 5-fluorouracil-induced encephalopathy. Jpn J Clin Oncol 2006;36:55-59.

15 Graham MA, Lockwood GF, Greenslade D, Brienza S, Bayssas M, Gamelin E: Clinical pharmacokinetics of oxaliplatin: a critical review. Clin Cancer Res 2000;6:1205-1218.

Table 1. Half-life of platinum-based agents

\begin{tabular}{lclll}
\hline & Oxaliplatin & Cisplatin & Carboplatin & Tetraplatin \\
\hline $\mathrm{t}_{1 / 2} \alpha, \mathrm{h}$ & $7.30 \pm 4.9$ & $0.22 \pm 0.15$ & $0.37 \pm 0.17$ & 0.16 \\
$\mathrm{t}_{1 / 2} \beta, \mathrm{h}$ & $239 \pm 54.4$ & $0.72 \pm 0.4$ & $1.93 \pm 0.23$ & 25.8 \\
\hline
\end{tabular}

